\title{
A Case Report of an Accessory and Cavitated Uterine Mass Treated with Total Laparoscopic Hysterectomy
}

\author{
Yukihiro Azuma," Fuminori Taniguchi," Hermawan Wibisono," Ai Ikebuchi," Maako Moriyama* and Tasuku Harada* \\ *Department of Obstetrics and Gynecology, Tottori University Faculty of Medicine, Yonago 683-8504, Japan
}

\begin{abstract}
Accessory and Cavitated Uterine Mass (ACUM) is a condition defined by the presence of a non-communicating uterine mass close to the insertion of the round ligament. ACUM is a rare Müllerian anomaly found in young women and is diagnosed by the presents of a central cavity in the uterus, lined by functional endometrium, and surrounded by a ring of smooth muscle. In most cases, surgical treatment is recommended due to severe dysmenorrhea. Herein, we present a case of a woman with severe dysmenorrhea since adolescence which was not relieved with any form of hormonal treatment. ACUM was suspected preoperatively based on MRI findings, and she was treated with total laparoscopic hysterectomy. A uterine mass was found at the insertion of the right round ligament during surgery. All her symptoms improved after resection.
\end{abstract}

Key words accessory and cavitated uterine mass; dysmenorrhea; fertile women; laparoscopic hysterectomy; müllerian anomaly

Müllerian anomalies can cause severe clinical symptoms and threaten quality of life. Congenital uterine anomalies may cause severe dysmenorrhea in adolescents and lead to problems with fertility. Approximately $7 \%$ of young women have an anatomical abnormality in their reproductive tract and the most frequent symptom of this is intolerable pain. ${ }^{1}$

Potter et al. reported a case of a rare Müllerian anomaly in young woman with a non-communicating, accessory uterine cavity adjacent to the normal uterus. ${ }^{2}$ Several cases with a similar accessory uterine cavity have been published previously, as isolated cystic adenomyomas or uterus-like masses. Acién et al. presented a novel hypothesis that juvenile and isolated cystic adenomyomas are different in origin from adenomyomas

Corresponding author: Fuminori Taniguchi, MD, $\mathrm{PhD}$ tani4327@tottori-u.ac.jp

Received 2021 February 2

Accepted 2021 February 25

Online published 2021 March 17

Abbreviations: ACUM, Accessory and Cavitated Uterine Mass; MRI, magnetic resonance imaging; TLH, total laparoscopic hysterectomy with internal cystic areas in adult women. The reports of non-communicating, accessory uterine cavities with cystic adenomyomas all have a common pathological finding: an Accessory and Cavitated Uterine Mass (ACUM) with normal, functional, endometrium. ${ }^{3}$ ACUM is found at the level of insertion of the round ligament and its origin may be liked to a dysfunction of the female gubernaculum. This congenital uterine malformation does not match the common Müllerian uterine anomalies found in the European Society of Human Reproduction and Embryology. ${ }^{4}$ ACUM is often misidentified as other uterine malformations (bicornuate uterus and segmental atresia), cystic adenomyoma, or degenerated leiomyomas.

The criteria used to diagnose ACUM are as follows: (a) an isolated, accessory, cavitated mass; (b) a normal uterus (with endometrial lumen), fallopian tubes, and ovaries; (c) surgical evidence with an excised mass and pathological finding; (d) an accessory cavity lined by endometrial epithelium, with glands and stroma; (e) chocolate-brown-colored fluid content; and (f) no adenomyosis. Most cases of ACUM have been diagnosed in women aged less than 30 years and those who are nulliparous; however, some cases have been reported in women who are older than 30 years or are multiparous. ${ }^{3}$ ACUM causes severe dysmenorrhea and recurrent pelvic pain in young women, usually requiring surgical treatment. Since most of these patients will wish to preserve fertility, excision of the tumor without hysterectomy is often the treatment of choice. Because of this, there are few reports of hysterectomy performed for ACUM; hence, we present a case of fertile woman with ACUM treated by total laparoscopic hysterectomy (TLH).

\section{PATIENT REPORT}

A 37-year-old woman visited our institution with complains of severe dysmenorrhea. She had regular menstrual cycles. Previously, she had undergone excision of a left ovarian endometrioma at another center when she was at 30 years old. After the surgery, she conceived spontaneously and had a normal vaginal delivery. However, the severe dysmenorrhea recurred after she resumed menstruating. Her symptoms were not relieved by any form of hormonal therapy, including 
A

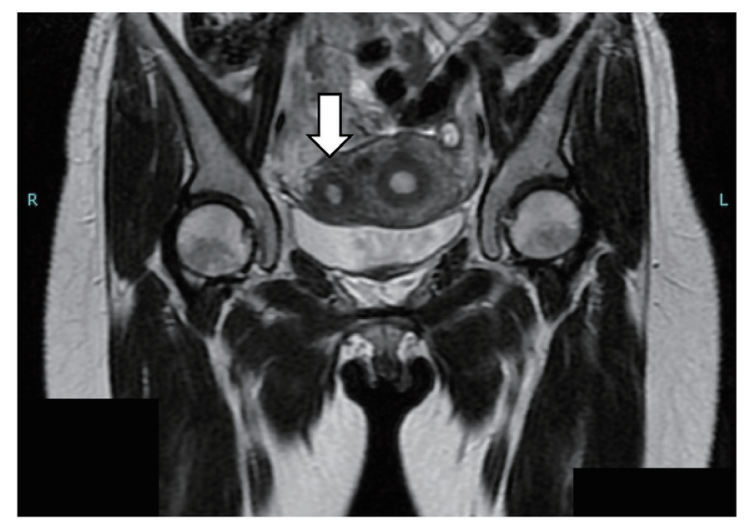

B

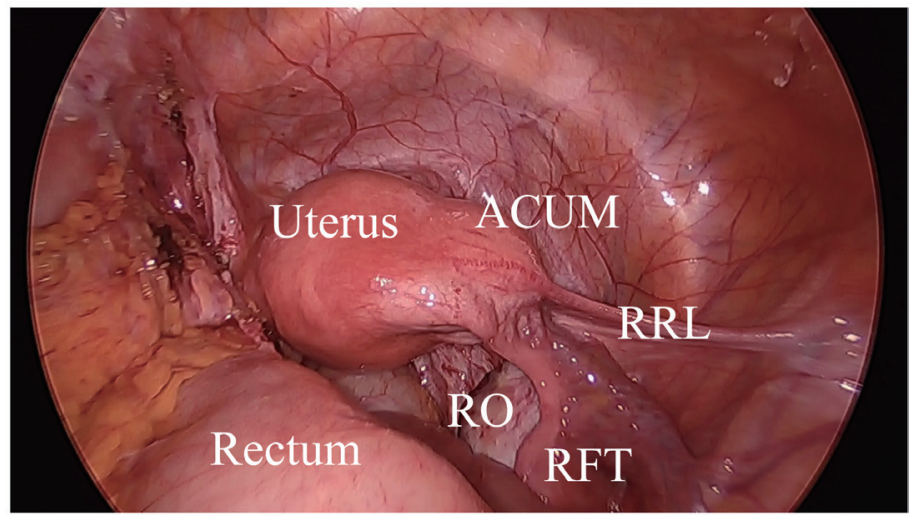

Fig. 1. ACUM lesion seen during laparoscopy. (A) Pelvic MRI (T2 weighted image, coronal plane) shows that the mass has a cavity with the blood component in the uterus close to the right round ligament (white arrow). (B) A focal lesion - the Accessory and Cavitated Uterine Mass (ACUM) - located in the myometrial wall at the level of insertion of RRL is seen. RFT, right fallopian tube; RO, right ovary; RRL, right round ligament.

low-dose estrogen-progestin combinations or progestin alone. Since she had no desire to conceive again, she elected to undergo TLH.

Transvaginal ultrasonography showed a normal sized uterus. A $27 \mathrm{~mm}$ mass, resembling fibroid, was observed in the right uterine wall. Pelvic MRI revealed that the mass had a cavity with the blood component in the uterus close to the right round ligament (Fig. 1A). No evidence of a fibroid or adenomyosis was detected. Drip infusion pyelography revealed no malformation of the urinary tract. All preoperative blood tests and physical examinations were normal.

The patient underwent TLH using a four-port approach after the establishment of pneumoperitoneum. We noted a focal lesion in the myometrial wall, at the right round ligament insertion (Fig. 1B). No adhesions existed around the lesion. The left adnexa was adherent to the retroperitoneum, due to the previous cystectomy. After identifying the bilateral ureters and uterine arteries, we cut off the parametrium. After incising the vaginal wall, we retrieved the whole uterus transvaginally. Finally, we closed the vaginal stump using sutures. The operation time was 168 minutes and blood loss was 10 $\mathrm{mL}$. No complications occurred. All the patient's symptoms were relieved completely.

The lesion seen to be isolated from the uterine cavity and no connection between the mass and right salpinx was observed macroscopically. The lesion contained a small round cyst filled with chocolate-colored fluid (Fig. 2A). Histological examination revealed a central cavity lined with functional endometrium and surrounded by smooth muscle (Fig. 2B). A pathologist determined that there was no evidence of adenomyosis.

\section{DISCUSSION}

We present a case of ACUM, which is a rare and poorly understood condition. Only 13 studies on ACUM have ever been published. There is a tendency to misdiagnose ACUM as a cystic adenomyoma. However, cystic adenomyomas consist of endometrial glands surrounded by endometrial-type stroma and smooth muscle but lack the uterus-like tissue found in ACUM. Acién et al. provided a few important points to bear in mind while suspecting an ACUM: the onset of symptoms, the recurrence of symptoms, the patient's age, an understanding of the pathology, and the most common locations of ACUMs. $^{5}$

Regarding the management of ACUM, it is important to diagnose the condition correctly before planning surgery. In this case, even though the patient had been suffering from painful menstruation since adolescence, she had not been diagnosed correctly before. We made the diagnosis of tumor, potentially a uterine myoma, based on ultrasonography. Peyron et al. described the utility of MRI in evaluating ACUM in 11 patients. ${ }^{6}$ MRI may facilitate timely diagnosis because the lesions are well defined with a central hemorrhagic cavity. In this case, a cavity filled with hemorrhagic fluid, distant from the uterine cavity, was found close to the right round ligament. MRI can therefore be beneficial as the first-line imaging modality in the diagnosis of uterine abnormalities.

Patients with ACUM experience severe dysmenorrhea refractory to conventional analgesics and hormonal therapies; hence, surgical treatment is indicated in almost all cases. Most patients with ACUM undergo tumor resection (and not hysterectomy) because of 
A

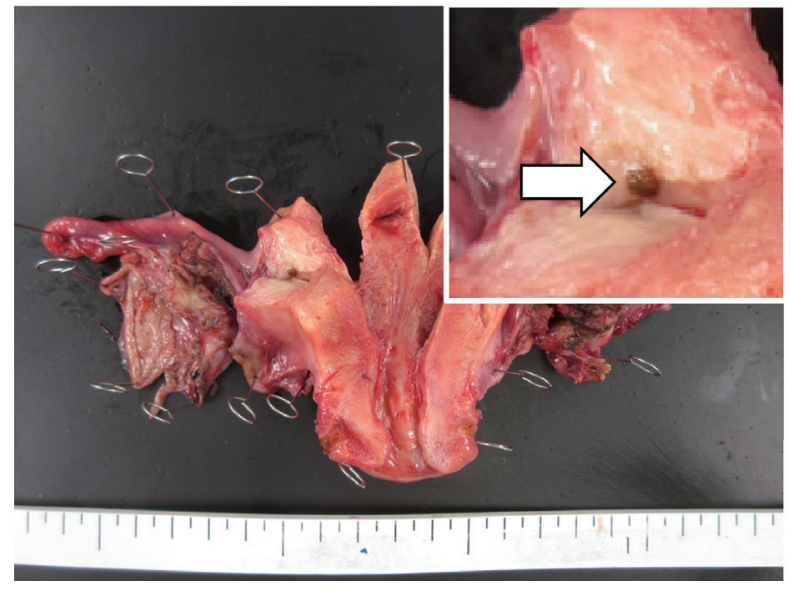

B

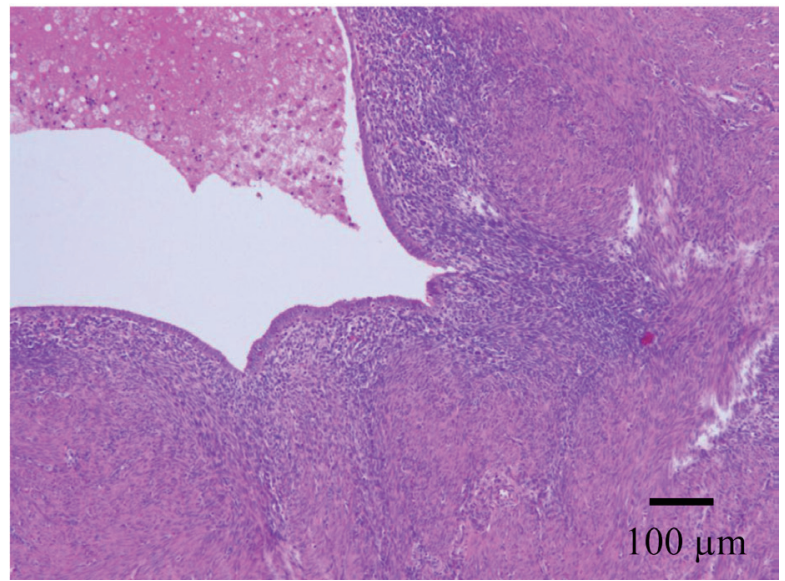

Fig. 2. Macroscopic and histological findings. (A) The lesion isolated from uterine cavity contains a small, round cyst filled with chocolate-colored fluid (white arrow). (B) The histological examination shows a central cavity lined with functional endometrium and surrounded by smooth muscle (hematoxylin and eosin staining). Bar $=100 \mu \mathrm{m}$.

a need to preserve their fertility. ${ }^{5,7}$ A hysterectomy should be recommended for patients who have no desire to conceive, because this can permanently relieve their dysmenorrhea. TLH is a common, safe, minimally invasive surgical treatment for benign gynecologic disease. However, it has been reported that urinary tract injuries occurred more often in laparoscopic hysterectomy than laparotomy. ${ }^{8}$ As Müllerian anomalies sometimes have urinary tract malformations, ${ }^{9}$ the surgeon should consider the possibility of this before planning a laparoscopic operation. We verified that there was no such malformation by a pyelography. In this case, both operation time and blood loss were within our expectations and we had no trouble during surgery. Consequently, we could relieve her from her symptoms completely. TLH is recommended for patients who suffer from ACUM as it is a safe surgical method offering permanent relief from symptoms.

Acknowledgments: We would like to thank Editage (www. editage.com) for English language editing.

The authors declare no conflict of interest.

\section{REFERENCES}

1 Dietrich JE, Millar DM, Quint EH. Obstructive reproductive tract anomalies. J Pediatr Adolesc Gynecol. 2014;27:396-402. DOI: 10.1016/j.jpag.2014.09.001, PMID: 25438708

2 Potter DA, Schenken RS. Noncommunicating accessory uterine cavity. Fertil Steril. 1998;70:1165-6. DOI: 10.1016/ S0015-0282(98)00380-X, PMID: 9848312
3 Acién P, Acién M, Fernández F, José Mayol M, Aranda I. The cavitated accessory uterine mass: a Müllerian anomaly in women with an otherwise normal uterus. Obstet Gynecol. 2010;116:1101-9. DOI: 10.1097/AOG.0b013e3181f7e735, PMID: 20966695

4 Grimbizis GF, Gordts S, Di Spiezio Sardo A, Brucker S, De Angelis C, Gergolet M, et al. The ESHRE/ESGE consensus on the classification of female genital tract congenital anomalies. Hum Reprod. 2013;28:2032-44. DOI: 10.1093/ humrep/det098, PMID: 23771171

5 Acién P, Bataller A, Fernández F, Acién MI, Rodríguez JM, Mayol MJ. New cases of accessory and cavitated uterine masses (ACUM): a significant cause of severe dysmenorrhea and recurrent pelvic pain in young women. Hum Reprod. 2012;27:683-94. DOI: 10.1093/humrep/der471, PMID: 22252088

6 Peyron N, Jacquemier E, Charlot M, Devouassoux M, Raudrant D, Golfier F, et al. Accessory cavitated uterine mass: MRI features and surgical correlations of a rare but under-recognised entity. Eur Radiol. 2019;29:1144-52. DOI: 10.1007/s00330-018-5686-6, PMID: 30159623

7 Peters A, Rindos NB, Guido RS, Donnellan NM. Uterinesparing Laparoscopic Resection of Accessory Cavitated Uterine Masses. J Minim Invasive Gynecol. 2018;25:24-5. DOI: 10.1016/j.jmig.2017.06.001, PMID: 28599883

8 Aarts JWM, Nieboer TE, Johnson N, Tavender E, Garry $\mathrm{R}, \mathrm{Mol} \mathrm{BWJ}$, et al. Surgical approach to hysterectomy for benign gynaecological disease. Cochrane Database Syst Rev. 2015;2015(8):CD003677. DOI: 10.1002/14651858.CD003677. pub5, PMID: 26264829

9 Acién P, Acién M. The presentation and management of complex female genital malformations. Hum Reprod Update. 2016;22:48-69. DOI: 10.1093/humupd/dmv048, PMID: 26537987 